\title{
Fundamental symmetries foundational to physics superspace
}

\section{Rowlands P.}

Department of Physics, University of Liverpool, Oliver Lodge Laboratory, Liverpool, UK;

E-mail: Rowlands <p.rowlands@liverpool.ac.uk>;

Symmetry is everywhere in Nature and has a particularly significant role in physics at the most fundamental level. Here, we propose that the origin of all the fundamental symmetries is in a Klein-4 group structure that connects the fundamental parameters mass, time, charge and space. The algebras associated with these parameters emerge in a sequence which first generates real numbers, then complex numbers, quaternions and multivariate vectors. The combined algebra has a special significance in being identical to that of the Dirac equation of relativistic quantum mechanics. This is the equation that applies to the point-like fermion, the most fundamental physical state. Many other symmetries and the fundamental symmetry-breaking that occurs between the four physical interactions can be seen to emerge from this foundational symmetric structure.

Keywords: symmetry, zero totality, Klein-4 group, nilpotent quantum mechanics, vacuum space.

DOI: $10.18698 / 2309-7604-2015-1-422-438$

\section{Introduction}

We are not very good observers - science is a struggle for us. But we have developed one particular talent along with our evolution that serves us well. This is pattern recognition. This is fortunate, for, everywhere in Nature, and especially in physics, there are hints that symmetry is the key to deeper understanding. And physics has shown that the symmetries are often 'broken', that is disguised or hidden. A classic example is that between space and time, which are combined in relativity, but which remain obstinately different.

Some questions are relevant here. Which are the most fundamental symmetries? Where does symmetry come from? How do the most fundamental symmetries help to explain the subject? Why are some symmetries broken and what does broken symmetry really mean? Many symmetries are expressed in some way using integers. Which are the most important?

We may begin our explanation with a philosophical starting-point. The ultimate origin of symmetry in physics is zero totality. The sum of every single thing in the universe is precisely nothing. Nature as a whole has no definable characteristic. Zero, in fact, is the only logical startingpoint. If we start from anywhere else we have to explain it. Zero is the only idea we couldn't conceivably explain.

To go from there it is convenient to give a semi-empirical answer, though it is possible to do it more fundamentally. The major symmetries in physics begin with just two ideas, duality and 
anticommutativity, and there are only two fundamental numbers or integers, 2 and 3. Everything else is a variation of these. In effect, anticommutativity is like creation, duality is like conservation. We can now start with a symmetry that is not well known, but which appears to be foundational to physics. This is between the four fundamental parameters

SPACE TIME MASS CHARGE

Here, mass has the more expansive meaning incorporating energy, and charge incorporates the sources of all 3 gauge interactions (electric, strong and weak). The symmetry-breaking between the charges is an emergent property, which we will show later emerges from algebra.

It is possible to represent the properties of these parameters symmetrically in terms of a Klein-4 group:

$\begin{array}{llll}\text { space } & \text { nonconserved } & \text { real } & \text { anticommutative } \\ \text { time } & \text { nonconserved } & \text { complex } & \text { commutative } \\ \text { mass } & \text { conserved } & \text { real } & \text { commutative } \\ \text { charge } & \text { conserved } & \text { complex } & \text { anticommutative }\end{array}$

Many physical, and even some mathematical, facts, not fully understood, may be seen principally as consequences of this symmetry. They include:

The conservation laws and Noether's theorem

The irreversibility of time

The unipolarity of mass

Why like charges repel but masses attract

The need for antistates

Lepton and baryon conservation and nondecay of the proton

Standard and nonstandard analysis, arithmetic and geometry

Zeno's paradox

The irreversibility paradox

Gauge invariance, translation and rotation symmetry 


\section{Representations of the parameter group}

One of the key aspects of the exactness of the symmetry between the parameters is that space, to be truly symmetrical to charge in its 3-dimensionality, is not just an ordinary vector, but one which has the properties of a Clifford algebra:

\begin{tabular}{|c|c|c|c|}
\hline $\begin{array}{lll}\mathbf{i} & \mathbf{j} & \mathbf{k}\end{array}$ & vector & & \\
\hline$i \mathbf{i} i \mathbf{j} i \mathbf{k}$ & bivector & pseudovector & quaternion \\
\hline$i$ & trivector & pseudoscalar & complex \\
\hline & scalar & & \\
\hline
\end{tabular}

The space-time and charge-mass groupings then become exact mirror images, 3 real +1 imaginary against 3 imaginary +1 real.

The vectors of physics are what Hestenes called multivariate vectors [1], isomorphic to Pauli matrices and complexified quaternions, with a full product

$$
a b=a \cdot b+i a \times b
$$

and a built-in concept of spin (which comes from the $i \mathbf{a} \times \mathbf{b}$ term). Hestenes showed, for example, that if we used the full product $\nabla \nabla \psi$ for a multivariate vector $\nabla$ instead of the scalar product $\nabla . \nabla \psi$ for an ordinary vector $\nabla$, we could obtain spin $1 / 2$ for an electron in a magnetic field from the nonrelativistic Schrödinger equation.

In the parameter group, space and time become a 4-vector with three real parts and one imaginary, by symmetry with the mass and charge quaternion, with three imaginary parts and one real.

$\begin{array}{cccc}\text { space } & \text { time } & \text { charge } & \text { mass } \\ \mathbf{i} x \mathbf{j} y \mathbf{k} z & \text { it } & \text { is je } \mathbf{k w} & 1 \mathrm{~m}\end{array}$

Vectors, like quaternions, are also anticommutative.

The group properties can be represented very simply using algebraic symbols for the properties / antiproperties: 


$\begin{array}{lrrr}\text { time } & -X & -y & Z \\ \text { charge } & X & -y & -Z \\ \text { space } & -X & y & -Z\end{array}$

In algebraic terms, this is a conceptual zero.

The symmetry may be assumed to be absolutely exact - no exception to this rule has ever been found in forty years [2-7]. And this condition can be used to put constraints on physics to derive laws and states of matter. We can also develop a number of representations, which not only show the absoluteness of the symmetry, but also the centrality to the whole concept of the idea of 3-dimensionality. A perfect symmetry between 4 parameters means that only the properties of one parameter need be assumed. The others then emerge automatically like kaleidoscopic images. It is, in principle, arbitrary which parameter we assume to begin with, as the following visual representations will show. The representations also suggest that 3-dimensionality or anticommutativity is a fundamental component of the symmetry.

In the colour representation, space, time, mass and charge, occupy concentric circles, divided into sectors suggesting the 3 properties / antiproperties. The properties (say, Real, Nonconserved, Discrete) can be by primary colours (say, Red, Green, Blue, or R, G, B), and the 'antiproperties' (Imaginary, Conserved, Continuous) by the complementary secondary ones (Cyan, Magenta, Yellow, or C, M, Y). All of these choices are arbitrary, and we can, for example, as in the second figure, reverse the representation. Only the overall pattern is fixed.
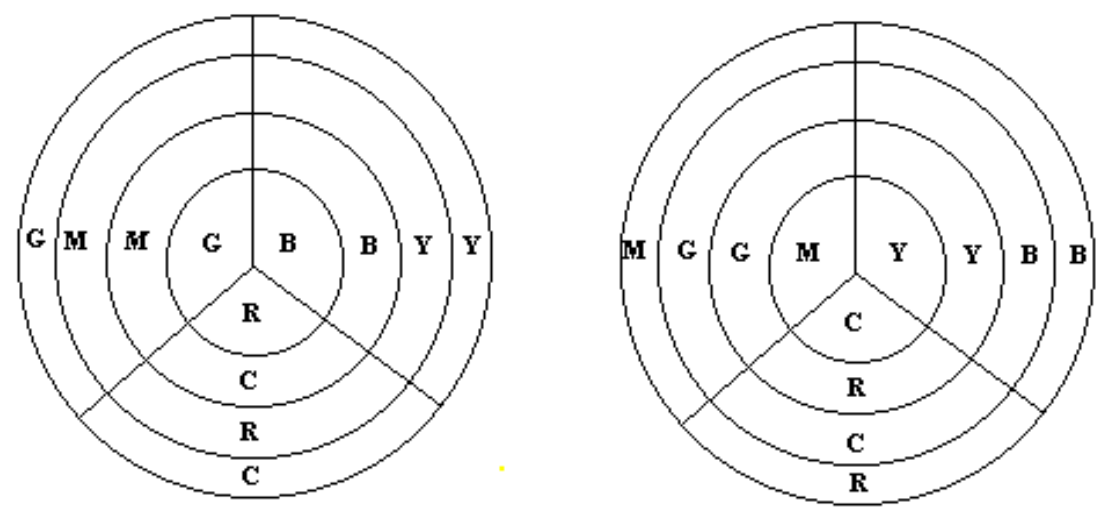

In any version of the representation, the total colour in each sector adds to white, representing zero. 


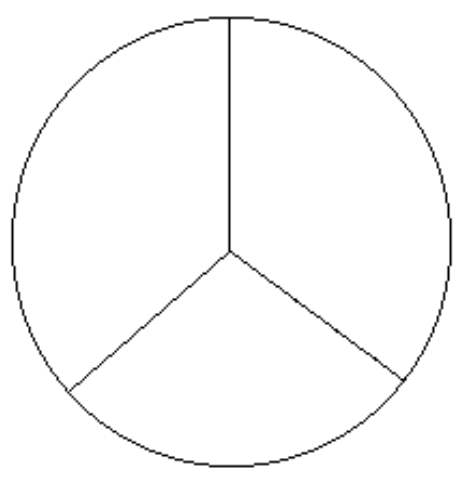

Alternatively, we can make a direct Cartesian plot of the $x, y$ and $z$ and $-x,-y,-z$ algebraic representations of properties and antiproperties from an origin at the centre of a cube to four of its corners. As in the colour representation, there is a dual version (here depicted using dotted lines), which can also be seen as a depiction of a dual group to that of the parameters with one of the properties / antiproperties reversed. Such a dual group emerges in the representation of the fundamental parameters in the Dirac equation.

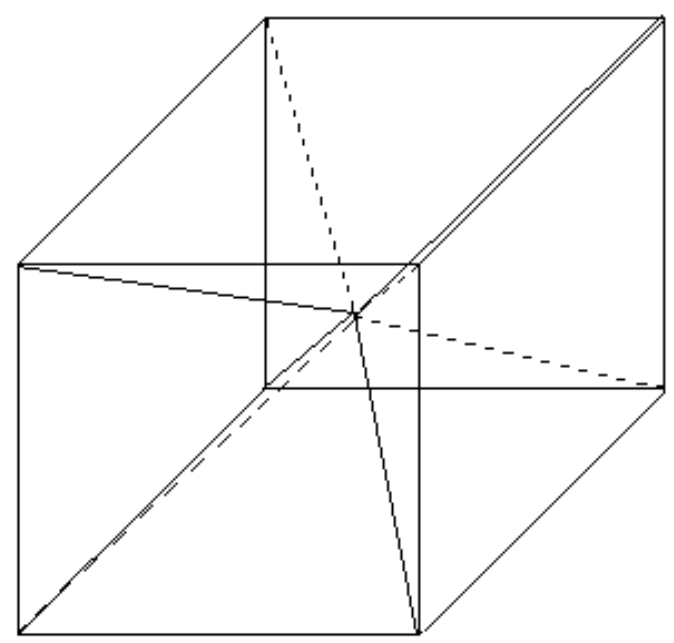

A third representation would place the parameters at the four faces or vertices of a regular tetrahedron, whose edges take on the primary / secondary colours as shown to stand for the six properties / antiproperties. There is, again, a built-in duality in this representation, as there is in the others. 


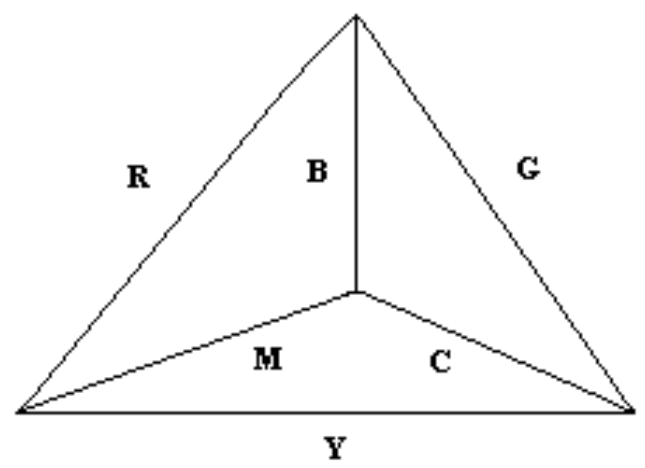

\section{Algebra and the parameters}

What is striking about the parameters and their properties is that they are purely abstract. They can be reduced, in effect, to pure algebra. Real / Imaginary and Commutative / Anticommutative are obviously so. But Conserved / Nonconserved can also be shown to be purely algebraic. They also each have their own algebra, which serves to define them. Their 'physical' properties come solely from this algebra.

$\begin{array}{lll}\text { Mass } & 1 & \text { scalar } \\ \text { Time } & \boldsymbol{i} & \text { pseudoscalar } \\ \text { Charge } & \boldsymbol{i} \boldsymbol{j} \boldsymbol{k} & \text { quaternion } \\ \text { Space } & \mathbf{i} \mathbf{j} \mathbf{k} & \text { vector }\end{array}$

The first three are subalgebras of the last, and combine to produce a version of it, let's say $\mathbf{i} \mathbf{j} \mathbf{k}$. In other words they are equivalent to a 'vector space', an 'antispace' to counter $\mathbf{i} \mathbf{j} \mathbf{~ k}$. We see why space appears to have a privileged status. It has 3 subalgebras:

bivector / pseudovector / quaternion, composed of:

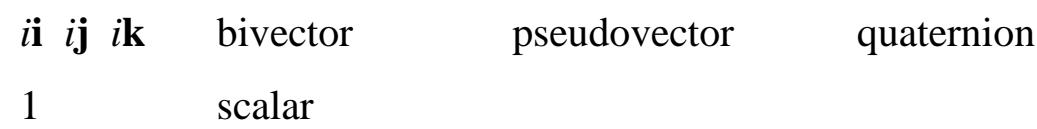

trivector / pseudoscalar / complex, composed of:

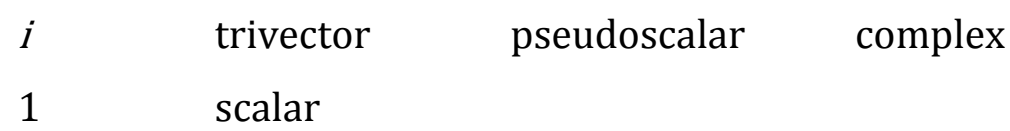


and scalar, with just a single unit:

$$
1 \text { scalar }
$$

The three parameters other than pace produce a combined vector-like structure, even though there is no physical vector quantity associated with them.

\begin{tabular}{|c|c|c|c|}
\hline mass & scalar & & 1 \\
\hline time & pseudoscalar & $i$ & 1 \\
\hline \multirow[t]{2}{*}{ charge } & quaternion & $i j k$ & 1 \\
\hline & $\begin{array}{l}\text { pseudovector } \\
\text { bivector }\end{array}$ & $\dot{i} \boldsymbol{j} \mathbf{j} \mathbf{k}$ & 1 \\
\hline COMBINED & vector & $\mathbf{i} \mathbf{j k}$ & $i j k i$ \\
\hline STRUCTURE & & $\mathbf{i} \mathbf{j k}$ & $\boldsymbol{i} \boldsymbol{j} \boldsymbol{j} \mathbf{k} i$ \\
\hline
\end{tabular}

This is what we will call vacuum space.

We now have another symmetry, leading to zero totality:

\begin{tabular}{|c|c|c|c|c|c|}
\hline \multirow[t]{4}{*}{ Space } & & & \multicolumn{3}{|c|}{ Everything else } \\
\hline & & & Mass & 1 & scalar \\
\hline & & & Time & $i$ & pseudoscalar \\
\hline & & & Charge & $i j k$ & quaternion \\
\hline Space & $\mathbf{i} \mathbf{j ~ k}$ & vector & Antispace & $\mathbf{i} \mathbf{j ~ k}$ & vector \\
\hline
\end{tabular}

We note that the algebras of charge, time, mass are subalgebras of vector algebra. It seems that, though all the parameters are equivalent in the group structure, they also produce a mathematical hierarchy, which suggests an 'evolutionary' structure in a logical, not a time sequence. This evolution can, in fact, be derived, and applied much more generally as a fundamental information process. It seems to operate in mathematics, computer science, chemistry and biology, as well as in more complex aspects of physics. 
We can also derive many aspects of the complexity directly. This is by packaging the physical information produced by combining the information from the individual algebras:

$\begin{array}{llll}\text { Time } & \text { Space } & \text { Mass } & \text { Charge } \\ \boldsymbol{i} & \mathbf{i} \mathbf{~ j ~ k} & 1 & \boldsymbol{i} \boldsymbol{j} \mathbf{k} \\ \text { pseudoscalar vector } & \text { scalar } & \text { quaternion }\end{array}$

Working out every possible combination of the four parameters and their 8 units requires 64 combined units. This turns out to be the algebra of the Dirac equation, the relativistic quantum mechanical equation of the fermion, the only true fundamental object that we know must exist. There 64 possible products of the 8 units are given by:

$\begin{array}{lcc}( \pm 1, \pm i) & 4 & \text { units } \\ ( \pm 1, \pm i) \times(\mathbf{i}, \mathbf{j}, \mathbf{k}) & 12 & \text { units } \\ ( \pm 1, \pm i) \times(\boldsymbol{i}, \boldsymbol{j}, \boldsymbol{k}) & 12 & \text { units } \\ ( \pm 1, \pm \boldsymbol{i}) \times(\mathbf{i}, \mathbf{j}, \mathbf{k}) \times(\boldsymbol{i}, \boldsymbol{j}, \boldsymbol{k}) & 36 & \text { units }\end{array}$

The + and - versions of the units:

\begin{tabular}{|c|c|c|c|c|c|c|}
\hline$i$ & $\tilde{J}^{*}$ & $k$ & $i \boldsymbol{i}$ & $i j$ & $i k^{*}$ & \\
\hline i & $\mathbf{j}$ & $\mathbf{k}$ & $\dot{\boldsymbol{n}}$ & $\dot{\boldsymbol{n}}$ & $i \mathbf{k}$ & \\
\hline $\mathrm{i} \boldsymbol{I}^{*}$ & $\mathbf{i} j$ & $\mathbf{i} k$ & $\dot{n} \dot{i}$ & $\dot{\boldsymbol{n}} \boldsymbol{j}$ & $\dot{n} k$ & \\
\hline $\mathrm{j} \boldsymbol{I}^{*}$ & $\mathrm{j} j$ & $\mathrm{j} k$ & $\hat{j} \boldsymbol{j} i$ & $\dot{j} j$ & $\dot{\jmath} k$ & \\
\hline $\mathrm{k} \boldsymbol{r}^{*}$ & $\mathrm{k} j$ & $\mathrm{k} k$ & $i \mathbf{k} i$ & $i \mathbf{k} j$ & $i \mathbf{k} i$ & \\
\hline
\end{tabular}

form a group. The simplest starting point for a group is to find the generators. These are the set of elements within the group that are sufficient to generate it by multiplication. Here they are marked with an asterisk.

Since vectors are complexified quaternions and quaternions are complexified vectors, we obtain an identical algebra if we use complexified double quaternions:

$\begin{array}{llllllll}i & j^{*} & k & i i & i j & i k^{*} & i & 1 \\ i & j & k & i i & i i & i k & & \end{array}$




$\begin{array}{llllll}i i^{*} & i j & i k & i i i & i i j & i i k \\ j i^{*} & j j & j k & i j i & i j j & i j k \\ k i^{*} & k j & k k & i k i & i k j & i k k\end{array}$

There is also a double vector version:

\begin{tabular}{|c|c|c|c|c|c|c|}
\hline $\mathbf{i}$ & $j^{*}$ & $\mathbf{k}$ & $\tilde{\boldsymbol{I}}$ & $\dot{j}$ & $i \mathrm{k}^{*}$ & $i$ \\
\hline $\mathrm{i}$ & $\mathbf{j}$ & $\mathbf{k}$ & $\tilde{\boldsymbol{n}}$ & $\tilde{\boldsymbol{n}}$ & $\mathbf{k}$ & \\
\hline $\mathrm{ii}^{*}$ & ij & $\mathbf{i k}$ & $\tilde{n} \mathbf{i}$ & $\ddot{i} \mathbf{j}$ & $i \mathbf{k}$ & \\
\hline $\mathrm{ji}^{*}$ & $\mathrm{jj}$ & $\mathrm{jk}$ & $j \mathrm{ji}$ & $\ddot{i j j}$ & $i j \mathrm{k}$ & \\
\hline $\mathbf{k i}^{*}$ & $\mathbf{k j}$ & $\mathbf{k k}$ & $\mathbf{k} \mathbf{i}$ & $\mathbf{k j}$ & $\mathbf{i k i}$ & \\
\hline
\end{tabular}

\section{The introduction of symmetry-breaking}

We started with eight basic units, but, by the time that we have worked out all the possible combinations of vectors, scalars, pseudoscalars and quaternions, we find that the Dirac algebra has 32 possible units or 64 if you have + and - signs. This group of order 64 requires only 5 generators. There are many ways of selecting these, but all such pentad sets have the same overall structure. However, the most efficient way of generating the $2 \times 32$ is to start with five composites, rather than eight primitives.

All the sets of 5 generators have the same pattern, as we can see by splitting up the 64 units into $1,-1, i$ and $-i$, and 12 sets of 5 generators, each of which generates the entire group:

\begin{tabular}{|c|c|c|c|c|c|c|c|c|c|}
\hline 1 & $i$ & & & & -1 & $-i$ & & & \\
\hline$\tilde{\boldsymbol{n}}$ & ij & $\mathbf{k}$ & $i k$ & $j$ & $-\tilde{\boldsymbol{I}}$ & $-\boldsymbol{i} \boldsymbol{j}$ & $-\mathbf{k}$ & $-i k$ & $-j$ \\
\hline$\tilde{\boldsymbol{I}}$ & $j$ & jk & $i i$ & $k$ & $-\dot{\boldsymbol{i}}$ & $-j$ & $-j \mathbf{k}$ & $-i j$ & $-i$ \\
\hline$k \mathbf{i}$ & $k j$ & $k \mathbf{k}$ & $i j$ & $i$ & $-k \mathbf{i}$ & $-k j$ & $-k \mathbf{k}$ & $-i j$ & $-i$ \\
\hline$\dot{a} i$ & $\ddot{\mathbf{n}} \boldsymbol{j}$ & $\ddot{n} k$ & $i \mathbf{k}$ & $\mathrm{j}$ & $-\dot{\boldsymbol{n}} \boldsymbol{i}$ & $-\tilde{\mathbf{n}} j$ & $-\tilde{i} k$ & $-i \mathbf{k}$ & $-j$ \\
\hline$\hat{j} \mathbf{i}$ & $\dot{\jmath} j$ & $\ddot{j} k$ & $\tilde{n}$ & $\mathbf{k}$ & $-\dot{j} \boldsymbol{i}$ & $-\tilde{\mathfrak{j}} \boldsymbol{j}$ & $-\dot{j} k$ & $-\hat{\boldsymbol{\Lambda}}$ & $-\mathrm{k}$ \\
\hline $\mathrm{k} i$ & $i \mathrm{k} j$ & $\mathrm{k} k \mathrm{k}$ & $\boldsymbol{j} \mathbf{j}$ & $\mathbf{i}$ & $-\mathbf{k} i$ & $-i \mathbf{k} j$ & $-\mathbf{k} k \mathbf{k}$ & $-\tilde{j}$ & $-\mathbf{i}$ \\
\hline
\end{tabular}

The creation of any set of 5 generators requires symmetry-breaking of one 3-D quantity. From the perfect symmetry of 
Proceedings of International Conference PIRT-2015

$\begin{array}{lllllllll}i & \mathrm{i} & \mathrm{j} & \mathbf{k} & 1 & \boldsymbol{i} & j & k\end{array}$

we rearrange to produce:

$\begin{array}{lllll}i & \mathrm{i} & \mathrm{j} & \mathrm{k} & 1 \\ i & & j & & k\end{array}$

and finally:

$$
\begin{array}{lllll}
i k & \mathbf{n} & \mathbf{j} & \mathbf{k} & 1 j
\end{array}
$$

The symmetry-breaking has an impact on the nature of the parameters involved. If we You have to break the symmetry of one space or the other, of $\mathbf{i}, \mathbf{j}, \mathbf{k}$ or $\boldsymbol{i}, \boldsymbol{j}, \boldsymbol{k}$. Since space is nonconserved and, therefore, rotation symmetric, we choose this to be charge. So, beginning with time, space, mass and charge, we may take one of each of $\boldsymbol{i}, \boldsymbol{j}, \boldsymbol{k}$ of charge on to each of the other three. Physically, to create the generators we have to distribute the charge units onto the other parameters. This creates new 'compound' (and 'quantized') physical quantities, which, using arbitrary names and symbols, we call 'energy', 'momentum' and 'rest mass'. So

$\begin{array}{cccc}\text { Time } & \text { Space } & \text { Mass } & \text { Charge } \\ \boldsymbol{i} & \mathbf{i} \mathbf{~ j ~ k} & 1 & \boldsymbol{i} \mathbf{j} k\end{array}$

become

$\begin{array}{lll}\text { Energy } & \text { Momentum } & \text { Rest Mass } \\ i & \mathbf{i} i \mathbf{j} \boldsymbol{i} \mathbf{k} \boldsymbol{i} & 1 \boldsymbol{j} \\ E & p_{x} p_{y} p_{z} & m\end{array}$

The combined object is nilpotent, squaring to zero, because

$$
\left(\mathbf{k} E+\mathbf{i} i p_{x}+\mathbf{j} i p_{y}+\mathbf{k} i p_{y}+j m\right)\left(\mathbf{k} E+\mathbf{i} i p_{x}+\mathbf{j} i p_{y}+\mathbf{k} i p_{y}+j m\right)=0
$$

We can identify this as Einstein's relativistic energy-momentum equation

$$
E^{2}-p^{2}-m^{2}=0
$$


or, in its more usual form,

$$
E^{2}-p^{2} c^{2}-m^{2} c^{4}=0
$$

\section{Nilpotent quantum mechanics}

The Dirac equation simply quantizes the nilpotent equation, using differentials in time and space, operating on a phase factor, for $E$ and $p$. So (1) becomes

$$
\left(\mp \boldsymbol{k} \frac{\partial}{\partial t} \mp i \boldsymbol{i} \nabla+\boldsymbol{j} m\right)( \pm \boldsymbol{i} \boldsymbol{k} E \pm \boldsymbol{i} \mathbf{p}+\boldsymbol{j} m) e^{-i(E t-\mathbf{p . r})}=0
$$

by simultaneously applying nonconservation and conservation. Here, we note there are four sign variations in $E$ and p. The fact that this is reduced by nilpotency from eight leads to another symmetry-breaking. We lose a degree of freedom, leading to chirality.

Written out in full the four components are:

$$
\begin{array}{ll}
(i k E+\dot{p}+j m) & \text { fermion spin up } \\
(i k E-\dot{p}+j m) & \text { fermion spin down } \\
(-i k E+\dot{p}+j m) & \text { antifermion spin down } \\
(-i k E-\dot{p}+j m) & \text { antifermion spin up }
\end{array}
$$

The signs are, of course, intrinsically arbitrary, but it is convenient to identify the four states by adopting a convention.

The spinor properties of the algebra still hold, even when we don't use a matrix representation, and $\psi$ is a 4-component spinor, incorporating fermion / antifermion and spin up / down states. We can easily identify these with the arbitrary sign options for the $i E$ and $\mathbf{p}$ (or $\sigma . p$ ) terms. This is accommodated in the nilpotent formalism by transforming $(i k E+i p+j m)$ into a column vector with four sign combinations of $i E$ and $\mathbf{p}$, which may be written in abbreviated form as $( \pm i \boldsymbol{k} E \pm \boldsymbol{i p}+\boldsymbol{j} m)$. Using an accepted convention, this can be either operator or amplitude. The symmetry between operator and amplitude is another leading to 0 . 


$$
( \pm \mathbf{k} E \pm i \mathbf{p}+j m)( \pm \mathbf{k} E \pm \dot{i}+j m) \rightarrow 0
$$

gives us both relativity and quantum mechanics - a version which is much simpler and seemingly more powerful than conventional quantum mechanics.

In quantum mechanics we take the first bracket as an operator acting on a phase factor. The $E$ and $\mathbf{p}$ terms can include any number of potentials or interactions with other particles. Squaring to 0 gives us the Pauli exclusion principle, because if any two particles are the same, their combination is 0 . In this form, we don't even need an equation, just an operator of the form $( \pm i k E$ $\pm \boldsymbol{i p}+\boldsymbol{j} m$ ) because the operator will uniquely determine the phase factor needed to produce a nilpotent amplitude. Rather than using a conventional form of the Dirac equation, we find the phase factor such that, using the defined operator,

$$
\text { (operator acting on phase factor })^{2}=\text { amplitude }^{2}=0 \text {. }
$$

If the operator has a more complicated form than that of the free particle, the phase factor will, of course, be no longer a simple exponential but the amplitude will still be a nilpotent. The same operation which gives us energy, momentum, and rest mass also gives us the broken symmetry between the 3 charges

$\begin{array}{lll}i & \mathbf{i} \boldsymbol{i} \mathbf{j} i \mathbf{k} \boldsymbol{i} & 1 \boldsymbol{j} \\ \text { weak } & \text { strong } & \text { electric }\end{array}$

which now adopt the characteristics of the mathematical objects they are connected to, and the corresponding group symmetries:

$\begin{array}{lll}\text { pseudoscalar } & \text { vector } & \text { scalar } \\ S U(2) & S U(3) & U(1)\end{array}$

The connections can be demonstrated with full rigour.

A particular subalgebra of the 64-part algebra creates a symmetry between the two spaces which remains unbroken. This is the $\mathrm{H} 4$ algebra, which can be obtained using coupled quaternions, with units $1, \boldsymbol{i i}, \boldsymbol{j} \boldsymbol{j}, \boldsymbol{k} \boldsymbol{k}$. The result is a cyclic but commutative algebra with multiplication rules

$$
\text { ii } i i=j j j=k k k=1
$$


Proceedings of International Conference PIRT-2015

$$
\begin{gathered}
i i j j=j j i i=k k \\
\ddot{j} k k=k k j j=i i \\
k k i i=i i k k=j j
\end{gathered}
$$

The same algebra can be achieved with the negative values of the paired vector units $1,-$ ii, $-\mathbf{j j}$, $-\mathbf{k k}$. ( 1 is equivalent here to $-i i$.) This time we have:

$$
\begin{aligned}
& (-\mathrm{ii})(-\mathrm{ii})=(-\mathrm{jj})(-\mathrm{j} \mathbf{j})=(-\mathbf{k k})(-\mathrm{kk})=1 \\
& (-\mathrm{ii})(-\mathbf{j} \mathbf{j})=(-\mathbf{j} \mathbf{j})(-\mathrm{ii})=(-\mathbf{k k}) \\
& (-\mathbf{j})(-\mathbf{k k})=(-\mathbf{k k})(-\mathbf{k k})=(-\mathbf{i}) \\
& (-\mathbf{k k})(-\mathrm{ii})=(-\mathrm{ii})(-\mathbf{k k})=(-\mathrm{jj})
\end{aligned}
$$

If we use the symbols $\mathbf{I}=\boldsymbol{i i}=-\mathbf{i i}, \mathbf{J}=\boldsymbol{j} \mathbf{j}=-\mathbf{j} \mathbf{j}, \mathbf{K}=\boldsymbol{k} \boldsymbol{k}=-\mathbf{k} \mathbf{k}, 1$, to represent this algebra, we can structure the relationships in a group table:

\begin{tabular}{|c|c|c|c|c|}
\hline$*$ & 1 & $\mathbf{I}$ & $\mathbf{J}$ & $\mathbf{K}$ \\
\hline 1 & 1 & I & J & $\mathbf{K}$ \\
\hline $\mathbf{I}$ & $\mathbf{I}$ & 1 & $\mathbf{K}$ & $\mathbf{J}$ \\
\hline $\mathbf{J}$ & $\mathrm{J}$ & $\mathbf{K}$ & 1 & $\mathbf{I}$ \\
\hline $\mathbf{K}$ & $\mathbf{K}$ & $\mathrm{J}$ & $\mathbf{I}$ & 1 \\
\hline
\end{tabular}

The group is a Klein-4 group, exactly like the parameter group.

All the standard aspects of spin and helicity are easily recovered with nilpotent quantum mechanics (NQM). This means that it is possible to find a spinor structure which will generate the NQM state vector. A set of primitive idempotents constructing a spinor can be defined in terms of the $\mathrm{H} 4$ algebra, constructed from the dual vector spaces:

$$
\begin{aligned}
& (1-i i-j j-\mathrm{kk}) / 4 \\
& (1-i i+j j+k k) / 4 \\
& (1+i i-j j+k k) / 4 \\
& (1+i i+j j-\mathrm{kk}) / 4
\end{aligned}
$$


As required the 4 terms add up to 1, and are orthogonal as well as idempotent, all products between them being 0 . The same terms can be generated using coupled quaternions rather than vectors:

$$
\begin{aligned}
& (1+i i+j j+k k) / 4 \\
& (1+i i-j j-k k) / 4 \\
& (1-i i+j j-k k) / 4 \\
& (1-i i-j j+k k) / 4
\end{aligned}
$$

The 'spaces' in the spinor structure are notably completely dual. The orthogonality condition effectively creates a quartic space structure with zero size, a point-particle.

\section{Vacuum}

Another way of looking at Pauli exclusion leads to another symmetry. Here, we say that Nature represents a totality of zero, and if you imagine creating a particle (with all the potentials representing its interactions) in the form

$$
( \pm \mathbf{k} E \pm \dot{\mathbf{p}}+j m)
$$

then you must structure the rest of the universe, so that it can be represented by

$$
-( \pm \mathbf{k} E \pm \mathbf{i p}+j m)
$$

The nilpotent formalism indicates that a fermion 'constructs' its own vacuum, or the entire 'universe' in which it operates, and we can consider the vacuum to be 'delocalised' to the extent that the fermion is 'localised'.

We can consider the nilpotency as defining the interaction between the localised fermionic state and the delocalised vacuum, with which it is uniquely self-dual, the phase being the mechanism through which this is accomplished. We can also consider Pauli exclusion as saying that no two fermions can share the same vacuum. The 'hole' left by creating the particle from nothing is the rest of the universe needed to maintain it in that state. We give it the name vacuum. So the vacuum for one particle cannot be the vacuum for any other. 
We can also think of the dual 'spaces' represented by $\mathbf{i}, \mathbf{j}, \mathbf{k}$ and $\mathbf{i}, \mathbf{j}, \mathbf{k}$ as combining together to produce zero totality in a point particle with zero size. It is the only way we can produce discrete points in space. The nilpotent formalism indicates that a fermion 'constructs' its own vacuum, or the entire 'universe' in which it operates, and we can consider the vacuum to be 'delocalised' to the extent that the fermion is 'localised'. We can consider the nilpotency as defining the interaction between the localised fermionic state and the delocalised vacuum, with which it is uniquely selfdual, the phase being the mechanism through which this is accomplished.

We can also understand the behaviour of fermion and vacuum in terms of more abstract mathematics. Set boundaries themselves have vanishing boundaries. The boundary of a boundary is zero:

$$
\partial \partial=\partial^{2}=0
$$

For $\mathrm{A}$ as subspace of the entire space $\mathrm{X}$, then the boundary $\partial \mathrm{A}$ is the intersection of the closures of $\mathrm{A}$ and of the complement of $\mathrm{A}$ or $\mathrm{X}-\mathrm{A}$, the closure being the union of the set and its boundary. Here the universe is $\mathrm{X}$, the fermion $\mathrm{A}$, the rest of the universe $\mathrm{X}-\mathrm{A}$. The point-fermion is itself a boundary. The boundary of the fermion is 0 . This is nilpotency.

\section{Vacuum space}

If we look at the four components of the fermion in (2) we see that two have $+E$ and two have $-E$. Where are those with $-E$ ? The answer is that they are in the vacuum space. There are as many antifermions as fermions. However, the chirality we have built into the structure (and that we can derive conventionally from the Dirac equation) means that only those in real space are observable.

If the lead term in the fermionic column vector, defines the fermion type, then we can show that the remaining terms are equivalent to the lead term, subjected to the respective symmetry transformations, $P, T$ and $C$, by pre- and post-multiplication by the quaternion units $\boldsymbol{i}, \boldsymbol{j}, \boldsymbol{k}$ defining the vacuum space:

$$
\begin{aligned}
& \text { Parity } \quad P \quad i( \pm i k E \pm \dot{p} \mathbf{p}+j m) i=( \pm i k E \quad \dot{p}+j m) \\
& \text { Time reversal } \quad T \quad k( \pm i k E \pm \dot{p} \mathbf{p}+j m) k=(i k E \pm i p+j m) \\
& \text { Charge conjugation } \quad C \quad-j( \pm i k E \pm i p+j m) j=\left(\begin{array}{ll}
i k E & i p
\end{array}+j m\right)
\end{aligned}
$$


We can easily show that $C P \equiv T, P T \equiv C$, and $C T \equiv P$ also apply, and that $T C P \equiv C P T \equiv$ identity as

$$
k(-j(i( \pm \mathbf{k} E \pm \dot{p} \mathbf{p}+j m) k) j) j=-k j i( \pm \mathbf{k} E \pm \dot{p}+j m) i j k=( \pm \mathbf{k} E \pm \dot{p} \mathbf{p}+j m)
$$

The nilpotent formalism defines a continuous vacuum $-( \pm \boldsymbol{i k} E \pm \boldsymbol{i p}+\boldsymbol{j} m)$ to each fermion state $( \pm i k E \pm \boldsymbol{i p}+\boldsymbol{j} m)$, and this vacuum expresses the nonlocal aspect of the state. However, the use of the operators $\boldsymbol{k}, \boldsymbol{i}, \boldsymbol{j}$ suggests that we can partition this state into discrete components with a dimensional structure. In fact, this is where the idempotents become relevant. If we postmultiply $( \pm i k E \pm \boldsymbol{i} \mathbf{p}+\boldsymbol{j} m)$ by the idempotent $\boldsymbol{k}( \pm \boldsymbol{i k} E \pm \boldsymbol{i} \mathbf{p}+\boldsymbol{j} m)$ any number of times, the only change is to introduce a scalar multiple, which can be normalized away.

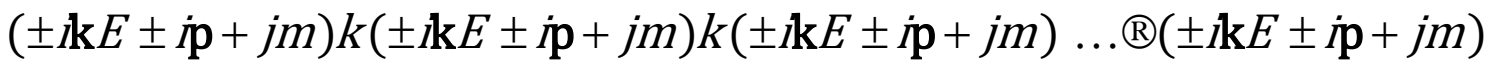

The identification of $\boldsymbol{i}(i \boldsymbol{k} E+\boldsymbol{i} \mathbf{p}+\boldsymbol{j} m), \boldsymbol{k}(\boldsymbol{i k} E+\boldsymbol{i} \mathbf{p}+\boldsymbol{j} m)$ and $\boldsymbol{j}(i \boldsymbol{k} E+\boldsymbol{i} \mathbf{p}+\boldsymbol{j} m)$ as vacuum operators and $(\boldsymbol{i k} E-\boldsymbol{i p}+\boldsymbol{j} m),(-i \boldsymbol{k} E+\boldsymbol{i} \mathbf{p}+\boldsymbol{j} m)$ and $(-i \boldsymbol{k} E-\boldsymbol{i p}+\boldsymbol{j} m)$ as their respective vacuum 'reflections' at interfaces provided by $P, T$ and $C$ transformations suggests a new insight into the meaning of the Dirac 4-spinor. We can now interpret the three terms other than the lead term in the spinor as the vacuum 'reflections' that are created with the particle. We can regard the existence of three vacuum operators as a result of a partitioning of the vacuum as a result of quantization and as a consequence of the 3-part structure observed in the nilpotent fermionic state, while the zitterbewegung can be taken as an indication that the vacuum is active in defining the fermionic state.

The operators $\boldsymbol{i}, \boldsymbol{j}, \boldsymbol{k}$ have many fundamental roles. They are charges, $C, P, T$ transformation operators, vacuum projections onto 3 axes, indicators of fermion / antifermion / spin up / down in the Dirac spinor, etc. They constitute the dimensions of vacuum space, dual to real space. The fermion has a half-integral spin because it requires simultaneously splitting the universe into two halves which are mirror images of each other at a fundamental level, but which appear asymmetric at the observational level because observation privileges the fermion singularity over vacuum. Zitterbewegung is an obvious manifestation of the duality, but, in observational terms, it privileges the creation of positive rest mass.

A particularly interesting example of the operation of vacuum space is reflection in a real mirror. This is due to an aspect of the electric force. The mirror produces a laterally-inverted virtual 
image. The mirror reflection is actually due to the rest of the universe ('vacuum') of which the mirror is a component. The virtual image is the reflection due to one component force. The mirror is constructed to concentrate the resources of vacuum almost entirely on this single force.

\section{Conclusion}

The Klein- 4 symmetry between mass, time, charge and space is the most fundamental in physics, and its algebraic representation allows us to generate a version of relativistic quantum mechanics which is applicable to the fundamental particle or fermionic state. Its group structure also generates the symmetry-breaking between the interactions which occurs at the most fundamental level in physics. Other symmetries which occur at the deepest levels in physics can be seen to be consequences of this one.

\section{References}

1. Hestenes, D. (1966). Space-Time Algebras, Gordon and Breach. New York: Gordon and Breach.

2. Rowlands, P. (1983). The fundamental parameters of physics. Speculat. Sci. Tech., 6, 69-80.

3. Rowlands, P. (2007). Zero to Infinity The Foundations of Physics. Singapore, London and Hackensack, NJ: World Scientific.

4. Rowlands, P. (2009). Are there alternatives to our present theories of physical reality?_arXiv, 0912.3433.

5. Rowlands, P. (2010). Physical Interpretations of Nilpotent Quantum Mechanics. arXiv: 1004.1523.

6. Rowlands, P. (2014). The Foundations of Physical Law. Singapore, London and Hackensack, NJ: World Scientific.

7. Rowlands, P. (2015). How Schrödinger's Cat Escaped the Box. Singapore, London and Hackensack, NJ: World Scientific. 